\title{
Turbulence structure near a sharp density interface
}

\author{
By IMAD A. HANNOUN \\ Jaycor, PO Box 85154, San Diego, CA 92138-9259, USA
}

HARINDRA J. S. FERNANDO

Mechanical and Aerospace Engineering, Arizona State University, Tempe, AZ 85287, USA

AND E. JOHN LIST

W. M. Keck Laboratory of Hydraulics and Water Resources, California Institute of Technology, Pasadena, CA 91125, USA

(Received 31 Oetober 1986 and in revised form 13 August 1987)

The effects of a sharp density interface and a rigid flat plate on oscillating-grid induced shear-free turbulence were investigated experimentally. A two-component laser-Doppler velocimeter was used to measure turbulence intensities in and above the density interface (with matched refractive indices) and near the rigid flat plate. Energy spectra, velocity correlations, and kinetic energy fluxes were also measured. Amplification of the horizontal turbulent velocity, coupled with a sharp reduction in the vertical turbulent velocity, was observed near both the density interface and the flat plate. These findings are in agreement with some previous results pertaining to shear-free turbulence near rigid walls (Hunt \& Graham 1978) and near density interfaces (Long 1978). The results imply that, near the density interface, the turbulent kinetic energy in the vertical velocity component is only a small fraction of the total turbulent kinetic energy and indicate that the effects of the anisotropy created by the density interface or the flat plate are confined to the large turbulence scales.

\section{Introduction}

As a consequence of a large number of geophysical and technological applications mixing in density-stratified fluids has recently received considerable attention. Development of turbulent wakes behind self-propelled bodies travelling in the pycnocline, growth of upper oceanic and lower atmospheric mixed layers into the thermocline and tropopause respectively, and the formation of clear-air turbulence in the atmosphere, are just a few examples where turbulent motions are dynamically affected by the ambient density stratification.

In the upper oceanic layers mixing occurs predominantly due to the turbulence generated by breaking surface waves, mean velocity shear, and surface cooling. Prior laboratory experiments studied these effects separately. In particular, the effects of breaking surface waves have been simulated by an oscillating grid (Kitaigorodski 1979), as it is known to have turbulence generating characteristics similar to breaking waves (Wang \& Wu 1985). Mixing in the presence of velocity shear can be simulated by driving the mixed layer over the non-turbulent layer using rigid rotating screens, by wind action, or by using specially designed pumps that create a differential mean velocity between two fluid layers. On the other hand, mixing due to surface cooling has been simulated by imposing an external buoyancy flux. 
Extensive discussion on the progress of research in this area is given in the reviews by Phillips (1977) and Turner (1979).

Previous attempts to estimate the mixing rate at a density interface in the absence of shear have been based on the energetics of the mixing process. Linden (1975) proposed that the rate of change of potential energy due to mixing is proportional to the total turbulent kinetic energy flux available at the entrainment interface. If the characteristic velocity scale near the interface is $u_{\mathrm{s}}$, this argument leads to a relationship of the form $u_{\mathrm{e}} / u_{\mathrm{s}} \sim R_{i \mathrm{o}}^{-1}$ where $u_{\mathrm{e}}$ is the rate of entrainment, $R_{i \mathrm{o}}=\Delta b D / u_{\mathrm{s}}^{2}$ is the overall Richardson number, $\Delta b$ is the buoyancy jump across the density interface and $D$ is the depth of the mixed layer. To obtain the time dependency of the mixed-layer depth Linden (1975) used $u_{\mathrm{s}} \sim\left(\sigma_{u}\right)_{0} \sim\left(\sigma_{w}\right)_{0}$, where $\sigma_{u}$ and $\sigma_{w}$ are r.m.s. horizontal and vertical velocities and the subscript $o$ refers to the quantity being measured in a homogeneous fluid at the same distance from the oscillating grid as the density interface. Linden also invoked the empirical relation obtained by Thompson \& Turner (1975) for the variation of $\sigma_{u}$ in a homogeneous fluid with distance from the grid $z$, i.e. $\sigma_{u} \sim z^{-\frac{3}{2}}$. However, other studies performed with oscillating grids strongly suggest that entrainment takes place at a rate much slower than predicted by the $u_{\mathrm{e}} /\left(\sigma_{u}\right)_{0} \sim R_{i}^{-1}$ relationship where $R_{i}=\Delta b D /\left(\sigma_{u}\right)_{0}^{2}$ Long (1978) proposed that this observation can be explained by considering the strong anisotropic nature of the turbulence near the density interface. Long extended the results of the rapid distortion theory of Hunt \& Graham (1978, referred to hereinafter as $\mathrm{HG}$ ) to estimate the vertical and horizontal r.m.s. velocities at the entrainment interface. Accordingly, large eddies of the size of the integral lengthscale of the turbulence tend to flatten at the density interface thus transferring the kinetic energy in the vertical velocity component to the horizontal components. Long further argued that the vertical r.m.s. velocity at the entrainment interface $w_{h}$ reduces to a value corresponding to that of the interfacial waves, which is much smaller than the r.m.s. horizontal velocity component near the entrainment interface. In a detailed analysis Long finds $w_{h} /\left(\sigma_{u}\right)_{0} \sim R_{i}^{-\frac{1}{4}}$ and $u_{\mathrm{e}} /\left(\sigma_{u}\right)_{0} \sim R_{i}^{-\frac{7}{4}}$. As pointed out by Fernando \& Long $(1985 a)$, the latter relationship can also be obtained by assuming that the rate of change of potential energy due to mixing is proportional to the vertical kinetic energy flux available at the interface. Experiments by Folse, Cox \& Schexnayder (1981) and Fernando \& Long (1983, 1985a,b) support $u_{\mathrm{e}} /\left(\sigma_{u}\right)_{\mathrm{o}} \sim R_{i}^{-\frac{7}{4}}$ behaviour, whereas Turner (1968) and Hopfinger \& Toly $(1976)$ find $u_{\mathrm{e}} /\left(\sigma_{u}\right)_{0} \sim R_{i}^{-\frac{3}{2}}$.

As evident from the above discussion the major emphasis of the existing theories for stratified shear-free turbulent mixing are the energy arguments. Some theories postulate that the rate of change of potential energy is proportional to the kinetic energy flux available at the interface, whereas Long (1978) conjectures that due to kinetic energy redistribution among the components near the density interface, the 'available energy for mixing' is much less than the total kinetic energy content near the interface. To establish the validity of these arguments a detailed investigation of the nature of turbulence near a density interface is needed, and in this paper, we report the details of such a study. This investigation also attempts to answer questions concerning the applicability of the rapid distortion theory in situations where there is no mean flow (e.g. Hunt 1984), and near sharp density interfaces.

In the present study, an oscillating grid was used to generate shear-free turbulence near a density interface and a two component laser-Doppler velocimeter (LDV) with index of refraction matching (\$2.4) was used to measure simultaneously the vertical component and one horizontal component of velocity in and near the entrainment 
interface and near a rigid flat plate. The results are compared with the theories of HG and Long (1978).

An attempt has been made earlier by McDougall $(1979 a, b)$ to study the turbulence characteristics near a density interface using LDV. However, only one component of velocity was measured at a time using an LDV that was reported to have a discontinuous signal. Furthermore, the Reynolds numbers based on the integral lengthscale and r.m.s. velocity used by McDougall (1979b) (about 40) were significantly lower than those in the present study (about 120). The present measurements include the vertical kinetic energy flux, velocity correlations and energy spectra.

\section{Experimental set-up}

The experiments described in this paper can be divided into three categories:

(i) turbulence measurements in a homogeneous fluid;

(ii) turbulence measurements in and near a density interface;

(iii) turbulence measurements near a rigid flat plate in a homogeneous fluid.

The experimental procedure in each of these experiments is discussed below.

\subsection{Tank and grid}

All experiments were carried out in a $3.35 \mathrm{~m}$ deep glass-walled tank with a horizontal cross-section of $1.15 \mathrm{~m} \times 1.15 \mathrm{~m}$. Other construction details of the tank are described in Papanicolaou (1985) and a schematic diagram of the experimental facility is shown in figure 1. The oscillating grid was placed in the upper portion of the tank and it consists of an array of rectangular bars $1.27 \mathrm{~cm}$ horizontal $\times 2.54 \mathrm{~cm}$ vertical. It is worth noting that rectangular bars have been chosen over square bars because they provide more structural strength for fixed grid solidity. The bars are placed on $6.35 \mathrm{~cm}$ centres thus yielding a grid solidity of $36 \%$. The oscillating grid is supported by a rigid frame suspended from the laboratory ceiling to minimize vibrations that may interfere with LDV measurements. A 3 h.p. d.c. motor is used to drive the oscillating grid via a reduction gear box. The rotary motion of the motor shaft is converted to the vertical reciprocating motion of the oscillating grid by using an eccentric wheel and sliders. The stroke of the grid motion was $6.35 \mathrm{~cm}$ with a fixed oscillation frequency of $2.25 \mathrm{~Hz}$. Due to the large size of the grid, a brace was needed to prevent excessive deformation of the grid. Four pieces of Plexiglas plate, each being $69.9 \mathrm{~cm} \times 15.2 \mathrm{~cm} \times 1.27 \mathrm{~cm}$, were glued together to form a $69.9 \mathrm{~cm} \times 69.9 \mathrm{~cm}$ open-ended square box. This box was glued to the top surface of the oscillating grid at a distance of $19.1 \mathrm{~cm}$ from each edge with the $15.2 \mathrm{~cm}$ side being vertical and the $1.27 \mathrm{~cm}$ side being placed directly on top of a grid bar. This resulted in a solid brace that did not alter the grid solidity. It is anticipated that the effect of the brace on the flow in the central parts of the tank is negligibly small.

\subsection{Laser-Doppler velocimeter system (LDV)}

A two-component LDV was used to measure the vertical and one horizontal velocity component simultaneously at the same point in the flow. The transmitting and receiving optics of the LDV are mounted on a counter-weighted instrument carriage. The carriage is attached to the tank and provides both vertical and lateral movement (normal to the plane of figure 1) for the instrumentation. The LDV employs a $7 \mathrm{~mW}$ $\mathrm{He}-\mathrm{Ne}$ laser (Hughes model 3227H-PC) and uses a two-reference-beam system. The general optical layout is shown in figure 1 and the details of the emitting optics are 


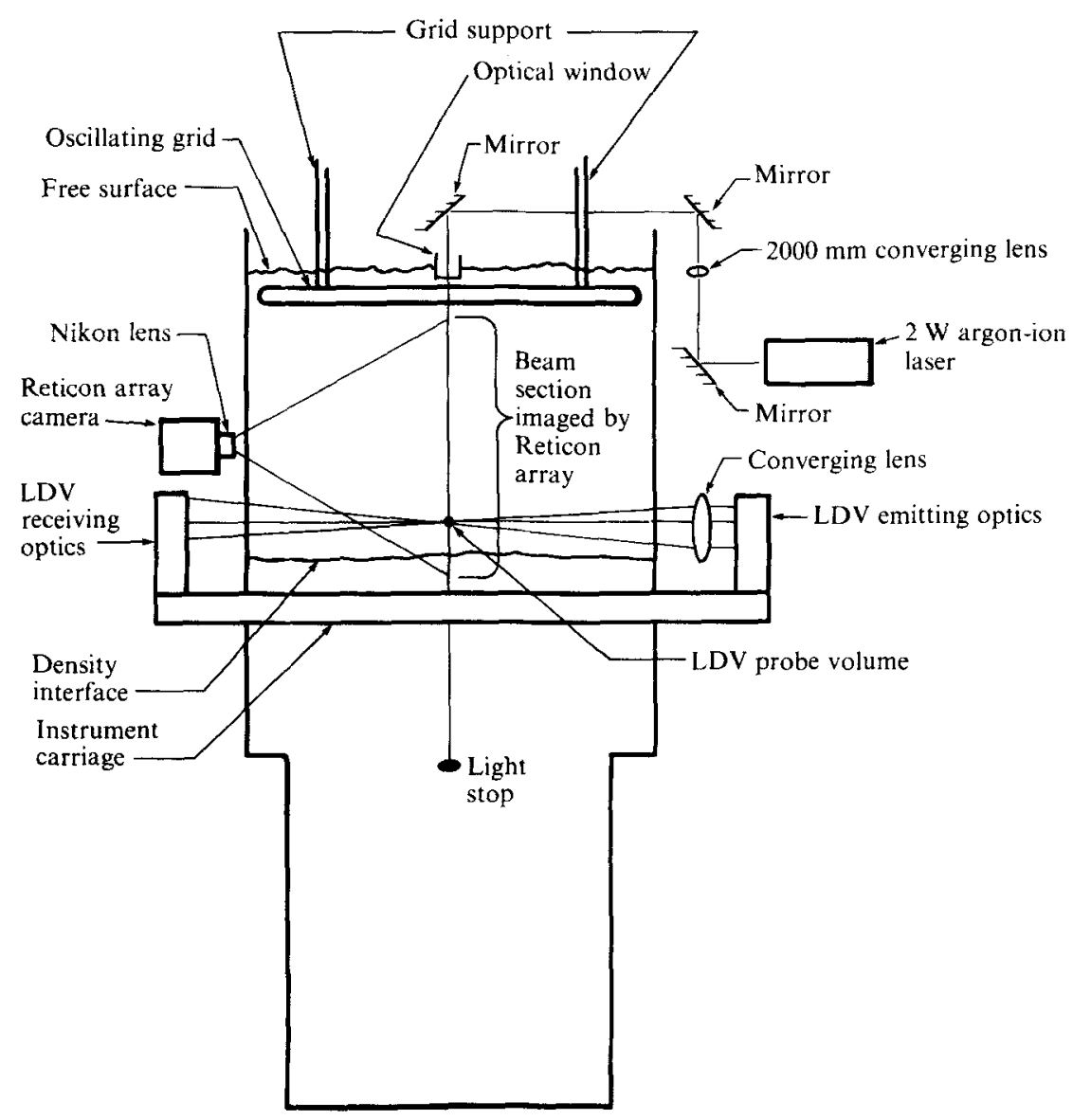

Figure 1. Schematic of experimental facility and optical layout.

shown in figure 2. The laser beams are split using prisms and are directed by a combination of mirrors and lenses to intersect in the middle plane of the tank. The frequencies of the laser beams are shifted in order to discriminate flow direction by using two $40 \mathrm{MHz}$ Bragg cells (Coherent model 305). The length of the probe volume is $3.6 \mathrm{~mm}$ for the vertical velocity component and $8.0 \mathrm{~mm}$ for the horizontal velocity component (Papanicolaou 1985). Other details of the optical set-up are given in Papanicolaou. The heterodyning light from the two reference beams and the scattering beam is focused on two fast response silicon photodiodes (EG\&G type DT-25) using two $12.5 \mathrm{~m}$ converging lenses.

The pre-amplified signal outputs of the photodiodes are fed to two identical laboratory-built signal processors. A schematic of one of the signal processors and the data acquisition system is shown in figure 3 . The raw signal from the photodiode is amplified and band-pass filtered (Krohn-Hite model 3202 filter) and the output signal is then fed to two parallel circuits: the first circuit is a zero crossing counter while the other parallel circuit is a validation circuit that re-initializes the counting if the signal falls below a certain preset threshold. This ensures that the only signals considered are due to particles passing in the central region of the probe volumes. The output of the signal processor is updated at a rate of about $200 \mathrm{~Hz}$. Details of the signal processor are discussed in Gartrell (1979). 




FIaUre 2. Schematic of LDV emitting optics. (a) Elevation. (b) Plan.

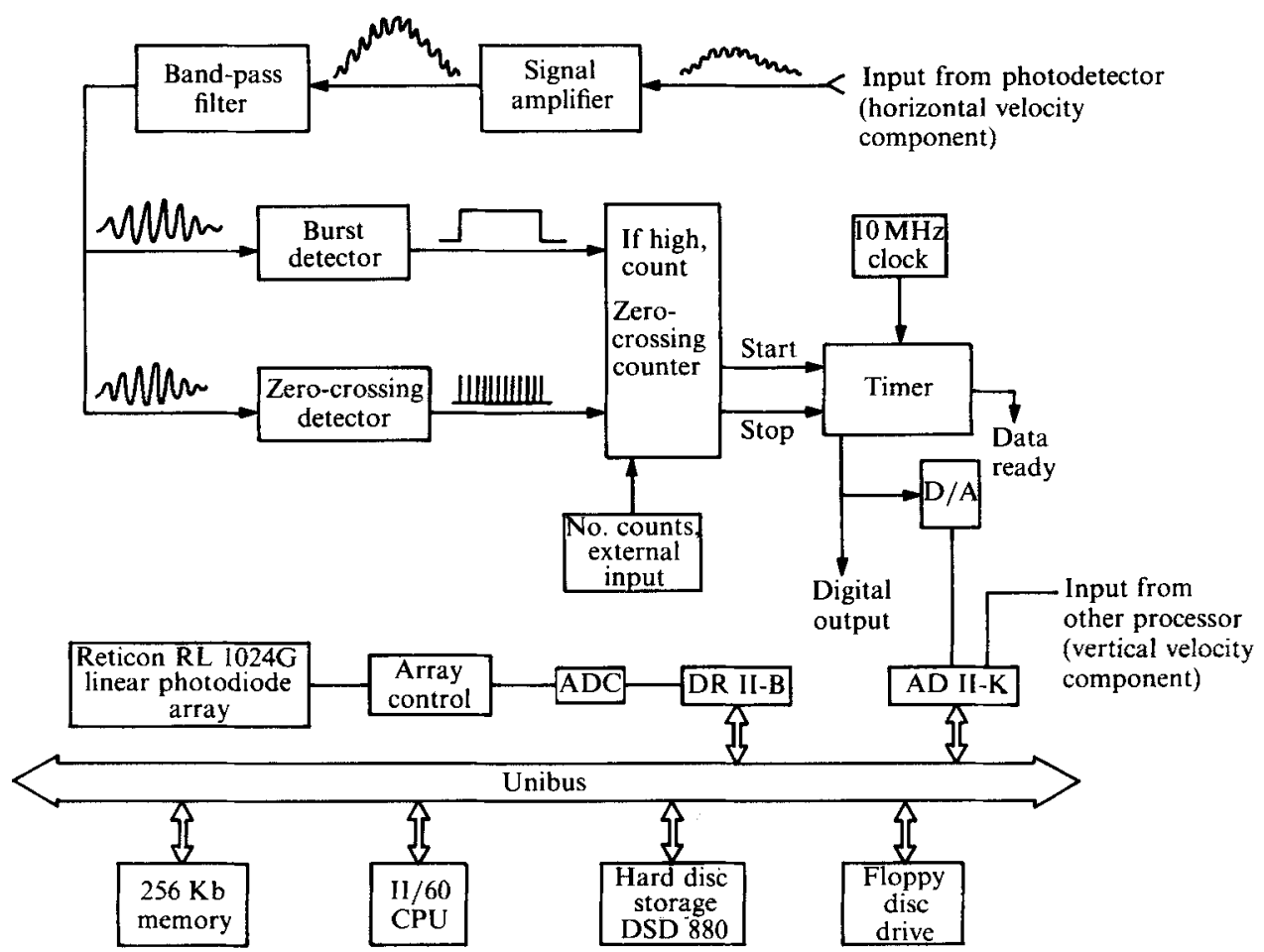

FIgure 3. Schematic of LDV processor, Reticon camera signal transmission, and layout of the data acquisition system.

\subsection{Monitoring of the interfacial position}

The position of the density interface was monitored using laser-induced fluorescence. This technique, as used in this study, is based on the ability of Rhodamine 6G dye to fluoresce at wavelengths near $570 \mathrm{~nm}$ when exposed to a laser beam having a wavelength of $514 \mathrm{~nm}$. For a range of dye concentrations, the intensity of the emitted light is proportional to the concentration of the dye. This provides a non- 
intrusive method for measuring concentrations. The details of the method of concentration measurement by laser-induced fluorescence will not be discussed here but a comprehensive discussion on this subject can be found in Liu et al. (1977). The $514 \mathrm{~nm}$ line of a $2 \mathrm{~W}$ argon-ion laser (Spectra Physics model 265) is directed vertically downward through a small glass window placed at the free surface as shown in figure 1 . Rhodamine $6 \mathrm{G}$ dye was premixed with the dechlorinated upperlayer fluid. A section of the laser beam was imaged onto an array of 1024 photodiodes (EG\&G Reticon model RL1024G) encased in an LC300A camera (EG\&G Reticon). The LC300A camera was rigidly mounted on a surveyor's tripod and used a Nikon $f 1.8$ lens. From the camera output, real-time concentration measurements were obtained along a straight line as illustrated in figure 1 . The data output rate from the LC300A camera was $20 \mathrm{~Hz}$ per pixel. The raw output of the photodiodes was corrected for beam attenuation as described by Koochesfahani (1984). The output was also corrected for optical imperfections, variable beam width, and lens aberration by performing a calibration that involved taking concentration measurements in a fluid with a uniform and known dye concentration as described in Papantoniou (1986). Although, in the experiments reported here, laser-induced fluorescence measurements were used for the sole purpose of defining the vertical position of the density interface in relation to the point where velocity measurements were made, this technique can also be used for detailed concentration measurements along a straight line. We have made such measurements and the results will be presented in a separate paper.

\subsection{Matching the refractive index}

It is well-known that index-of-refraction fluctuations create problems with LDV measurements. The consequences of these undesirable effects have been studied by McDougall $(1979 a)$. He concluded that, in general, the index-of-refraction fluctuations induce 'pseudoturbulence' in the high-frequency spectrum. To overcome this problem McDougall used two different solutes (sugar and Epsom salt) to obtain two fluids with different densities but with matching refractive indices. Owing to the fact that our tank is more than eighty times the volume of that used by McDougall, use of Epsom salt is not economical. Moreover, the choice of solutes is very limited in order to avoid the phenomenon of double diffusive convection, which introduces a stringent constraint on solute selection. After an exhaustive search, we have been successful in determining that the refractive indices can be matched while maintaining a significant density difference (up to $2.0 \%$ ) by using ethyl alcohol and common salt as solutes in the upper and lower layers respectively. In this way, the phenomenon of double diffusive convection is overcome altogether since both solutes are stably stratified. In any event, no double diffusion of the salt-finger type was observed in any of the experiments. Furthermore, alcohol and salt are quite inexpensive and their molecular diffusivities are approximately equal. A hand refractometer (American Optics model AO 10419) was used to match the refractive indices of the two layers. The tank was covered by a sheet of plastic in order to minimize alcohol evaporation, which was observed to be negligible after the cover was installed. Details of the method of matching the refractive indices using salt and alcohol are in Hannoun (1985).

\subsection{Procedure for density-stratified experiments}

Prior to the start of an experiment involving density stratification two fluid volumes were prepared with salt and alcohol as solutes respectively. After matching the refractive indices and ensuring that the temperature difference between the layers 
was less than $0.1 \mathrm{~K}$ the aqueous alcohol solution (typically $2-3 \%$ alcohol by weight) was allowed to spread very slowly over the lower saline layer using a carefully designed manifold. The manifold consists of a perforated horizontal pipe placed at the top of the lower layer. The alcohol solution was fed to the pipe under gravity, and the fluid forced out from the perforations formed a thin sheet and spread over the heavy layer. To avoid interfacial mixing, the filling process proceeded at a very slow rate and it took several hours to stratify the tank.

In the two-layer stratified mixing experiments the depth of the upper mixed layer is a slowly increasing function of time. In such experiments, the velocities were measured at a fixed point in space while the interface was moving downward. Hence, measuring velocities at different times is equiyalent to sampling velocities at different distances away from the density interface. During the duration of an experiment the maximum variation in $D$ was limited to $10 \%$ of its original value, thus the effect of the interfacial movement on the overall Richardson number $R_{i}$ and other parameters is small. Velocity measurements were not started until the upper layer grew by at least $20 \%$ of its depth after the end of the stratification process. This was done in order to minimize the effects of any anomalies from the tank filling process.

\subsection{Flate-plate experiments}

Flat-plate experiments were performed in a homogeneous fluid, the fluid being filtered tap water. The flat plate consisted of a $115 \mathrm{~cm} \times 10.0 \mathrm{~cm} \times 1.27 \mathrm{~cm}$ Plexiglas plate. The plate was placed in a position parallel to the grid at a distance of $21.2 \mathrm{~cm}$ away from the lowest point of the grid. At this position the width of the plate was about five times the integral lengthscale of the turbulence. Since the velocities were measured in a plane equidistant from the edges of the plate, effects due to the finite width of the plate can be considered to be minimal.

\subsection{Data acquisition and processing}

The analog output of the LDV processor was fed to an AD11-K analog-to-digital converter and stored in the memory of a Digital PDP 11/60 computer as illustrated in figure 3. Sampling rates were controlled by an external trigger and were fixed to $25 \mathrm{~Hz}$ per velocity component. No mechanism was used to ensure the arrival of both velocity signals at the same time. However, the new data update rate was about $200 \mathrm{~Hz}$. As a result, we can deduce that both components of velocity are measured within about $0.005 \mathrm{~s}$. This should not have a significant effect on the velocity correlations.

The analog output of the photodiode array was digitized using a custom built analog-to-digital converter (Papantoniou 1986) and was then fed to the PDP 11/60 computer through a DMA interface. The data were recorded on a DSD 880 Winchester disc. Each pixel was sampled at a rate of $20 \mathrm{~Hz}$ when concentration data were being recorded. Other details of the array data acquisition system are in Papantoniou (1986). Owing to the large amount of data generated and the finite disc storage volume we were limited to sampling concentrations before the beginning and at the end of velocity measurements. Each of the concentration sampling intervals lasted $90 \mathrm{~s}$. From these two concentration records the mean position of the interface was determined at two distinct time intervals. Intermediate interfacial positions were obtained by interpolation according to the relationship $u_{\mathrm{e}} /\left(\sigma_{u}\right)_{\mathrm{o}} \sim R_{i}^{-\frac{3}{2}}$. The concentration and velocity measurements were transferred to Digital PDP 11/24 and VAX 750 computers where all data analysis and reduction was performed. 


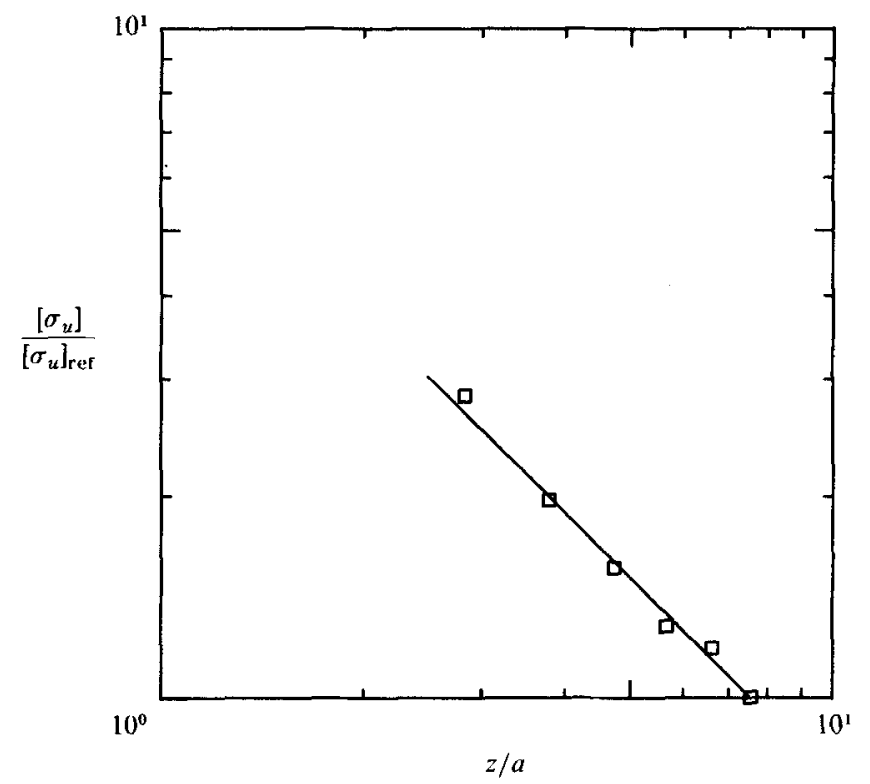

Figure 4. Variation of the non-dimensional r.m.s. horizontal velocity with non-dimensional distance away from the grid. $\left[\sigma_{u}\right]$ is normalized by its value at $z / a=7.7 . \square$, experimental results. - - least square fit to the data of the form $\left[\sigma_{u}\right] \sim z^{-1}$.

\section{Experimental results}

\subsection{Measurements in a homogeneous fuid}

In this section we will briefly present turbulent velocity measurements in a homogeneous fluid. Although other authors have already reported similar measurements we feel that these results constitute an important reference for comparison with measurements across a density interface and near a rigid flat plate. Moreover, we report here the values of the velocity correlation coefficients, kinetic energy fluxes, and spectra, which have not been previously measured. In figure 4 the measured r.m.s. horizontal turbulent velocities $\dagger,\left[\sigma_{u}\right]$, are plotted in non-dimensional form against $z / a$, where $z$ is a distance away from a virtual origin coinciding with the lowest position of the grid and $a$ is the amplitude of grid oscillation. The virtual origin was determined from the ordinate intercept when $z_{\mathrm{a}}$, a vertical distance away from an arbitrary origin, is plotted against $1 /\left[\sigma_{u}\right]$ and a least-square line is fitted to the data. It can be seen from figure 4 that a law of the form $\left[\sigma_{u}\right] \sim 1 / z$ satisfactorily describes the data. This is in agreement with Hopfinger \& Toly (1976), with the exception that the virtual origin in this study is about $3 \mathrm{~cm}$ away from the value predicted by them. This discrepancy may be due to the slight difference in grid geometry since rectangular bars were used in this study as opposed to the square bars used by them. It is also worth noting that the virtual origin for the vertical r.m.s. velocity, $\left[\sigma_{w}\right]$, obtained in a similar fashion, is within $1 \mathrm{~mm}$ of the virtual origin for $\left[\sigma_{u}\right]$. Moreover $\left[\sigma_{w}\right]$ also satisfies a law of the form $\left[\sigma_{w}\right] \sim 1 / z$. A spatial averaging procedure was used to compensate for measured variation of $\sigma_{u}$ and $\sigma_{w}$ in a

$\dagger$ Throughout this paper, the brackets [ ] represent a spatial average in a horizontal plane along a straight line. In all homogeneous fluid measurements without the rigid plate thirteen points in the central region of the tank separated by $1 \mathrm{~cm}$ were used for the averaging. Also, an overbar denotes time averaging. 


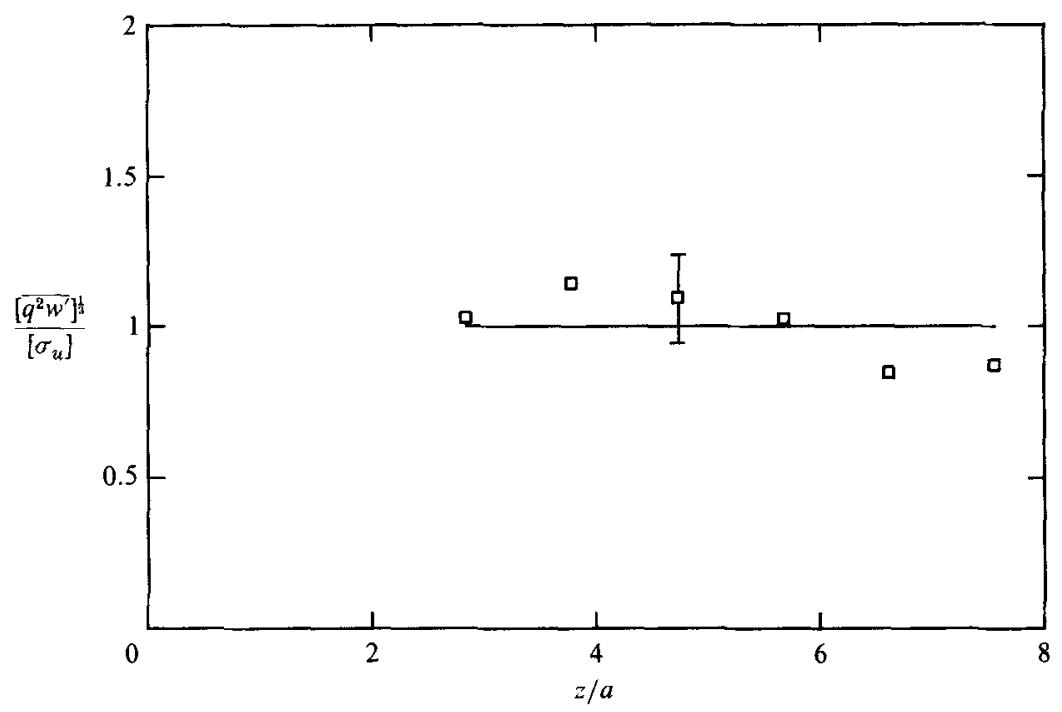

Figure 5. Variation of $\left[\overline{q^{2} w^{\prime}}\right]^{\frac{1}{3}} /\left[\sigma_{u}\right]$ with non-dimensional distance away from the grid $\square$, experimental results.

horizontal plane. The variation in $\sigma_{u}$ in a horizontal plane has a standard deviation of $5 \%$ of $\left[\sigma_{u}\right]$ at $z / a=6.60$. McDougall $(1979 b)$ has earlier reported on this inhomogeneity in velocity in a horizontal plane. The spatially-averaged correlation coefficient, $\left[\overline{u^{\prime} w^{\prime}} / \sigma_{u} \sigma_{w}\right]$, where $u^{\prime}$ and $w^{\prime}$ are fluctuating horizontal and vertical velocities respectively, is -0.023 with a standard deviation of 0.07 at $z / a=6.60$. The average value of $\left[\sigma_{w}\right] /\left[\sigma_{u}\right]$ is 1.32 for six values of $z / a$ ranging from 2.80 to 7.60 . This is slightly higher than the value of 1.2 measured by Hopfinger \& Toly (1976).

From simultaneous measurements of $u^{\prime}$ and $w^{\prime}$ at the same point, we can obtain the vertical kinetic energy per unit mass flux term $\left[\overline{q^{2} w^{\prime}}\right]$, where $q^{2}=\frac{1}{2}\left(u^{\prime 2}+v^{\prime 2}+w^{\prime 2}\right)$. In doing so we make an additional assumption that $\left[\overline{v^{\prime 2} w^{\prime}}\right]=\left[u^{\prime 2} w^{\prime}\right]$ where $v^{\prime}$ is the fluctuating horizontal velocity component in a direction normal to that of $u^{\prime}$. This assumption is needed since we do not measure $v^{\prime}$ and it is justified on the basis that there is no apparent reason why the time-averaged statistics in the direction of $v^{\prime}$ should differ from those in the direction of $u^{\prime}$ considering the one-dimensional nature of the turbulent energy propagation and the geometrical configuration of the apparatus. Accordingly, we will take $q^{2}=\frac{1}{2}\left(2 u^{\prime 2}+w^{\prime 2}\right)$. Hopfinger \& Linden (1982) have assumed a relationship of the form $\overline{q^{2} w^{\prime}} \sim \sigma_{u}^{3}$ for turbulence generated by an oscillating grid. To test the validity of such a relationship we plot $\left[\overline{q^{2} w^{\prime}}\right]^{\frac{1}{3}} /\left[\sigma_{u}\right]$ versus $z / a$ as shown in figure 5 . From figure 5 it seems that $\left[\overline{q^{2} w^{\prime}}\right]^{\frac{1}{3}} /\left[\sigma_{u}\right]$ is a constant independent of $z / a$ if we take into account that the estimated error in $\left[\overline{q^{2} w^{\prime}}\right]$ is about $15 \%$ of its value.

The integral timescale of the turbulence $\tau$ defined as the area under the vertical velocity autocorrelation coefficient curve, was found to be well represented by a function of the form $[\tau] \sim z^{2}$. Thus, if we define the integral lengthscale of the turbulence as $L=[\tau]\left[\sigma_{w}\right]$, then we obtain $L=0.1 z$ for our experiments. In figure 6 , we present an energy spectrum for the vertical velocity fluctuations at $z / a=6.60$. It is possible to observe a range where the spectrum decays as $f^{\frac{5}{3}}$ which seems to follow a prediction of the spectral decay in the inertial subrange by Tennekes (1975) in the absence of a mean flow. 


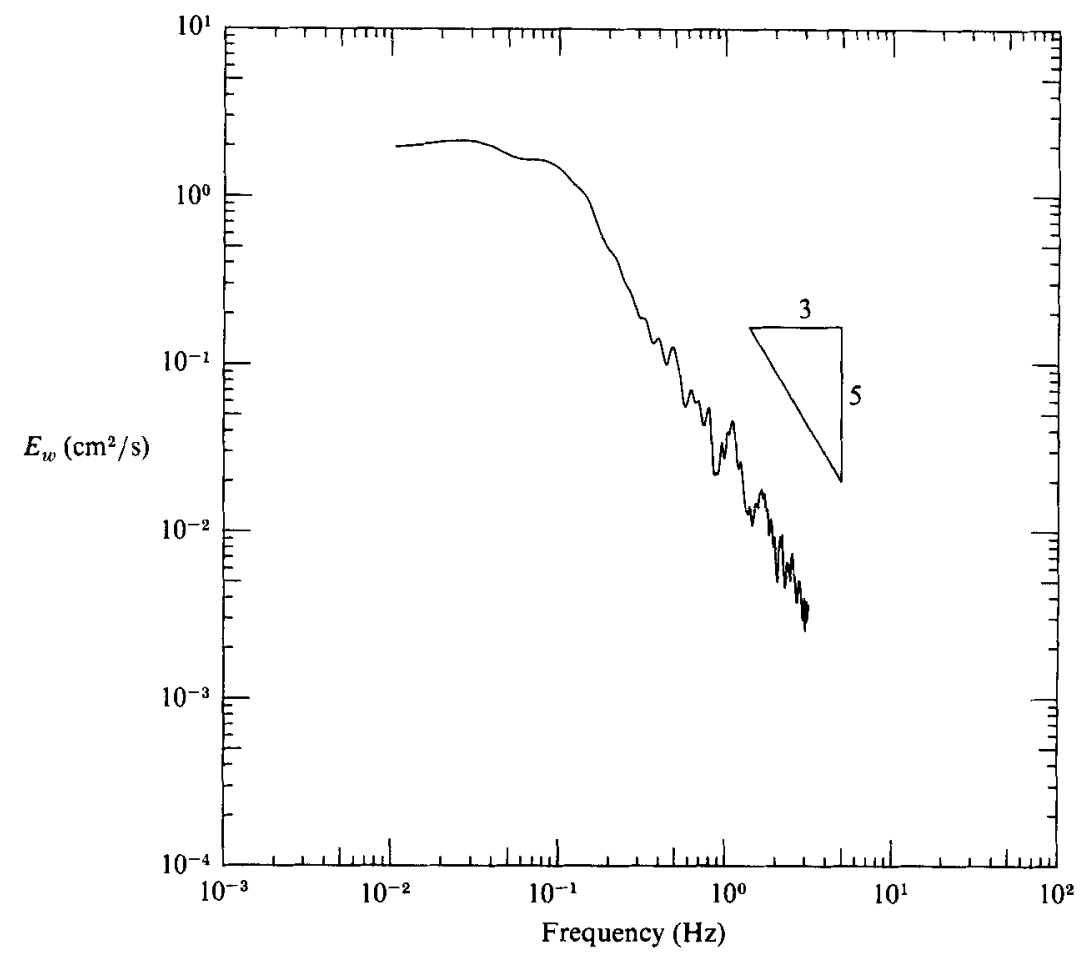

FIgure 6. Energy spectrum for the vertical velocity fluctuations in a homogeneous fluid.

$$
z / a=6.60 \text {. }
$$

\subsection{Measurements in density stratified fluids}

As discussed in $\$ 2.5$ the velocity measurements in the experiments with a density interface were performed at a fixed point in space with the interface migrating very slowly. The time trace of velocity is divided into several segments and the position of the interface relative to the measuring volume is calculated for each segment by interpolation as pointed out in $\$ 2.5 \dagger$. In figures $7(a)$ and $7(b)$ we plot normalized values of $\sigma_{u}$ and $\sigma_{w}$, the horizontal and vertical r.m.s. components respectively, as a function of $\xi / L_{0}$ where $\xi$ is a vertical distance measured from the density interface and $L_{\mathrm{o}}$ is the integral lengthscale of the turbulence measured in a homogeneous fluid at the vertical location of the interface. From both figures $7(a)$ and $7(b)$, which correspond to two different values of the stability parameter $R_{j}$, where $R_{j}=$ $\Delta b / L_{\mathrm{o}} /\left[\sigma_{u}\right]_{0}^{2}$, we notice that $\sigma_{u} /\left[\sigma_{u}\right]_{0}$ is amplified whereas $\sigma_{w} /\left[\sigma_{w}\right]_{0}$ shows a monotonic decay as the interface is approached. These measurements indicate that there is a transfer of energy from the vertical component to the horizontal components thus rendering the turbulence strongly anisotropic. It is also apparent from figures $7(a)$ and $7(b)$ that the effects of the density interface extend to about $\xi / L_{0} \sim 1$. Figure $8(a)$ shows the energy spectra for the vertical velocity fluctuations $E_{w}$ for various non-dimensional distances $\xi / L_{\mathrm{o}}$ away from the density interface, whereas figure $8(b)$ gives the energy spectra for the horizontal velocity fluctuations. From figure $8(a)$ we observe that there is preferential attenuation of low frequencies (large scales) as we approach the interface implying that the density interface has a

$\dagger$ The position of the interface for each data segment is defined as the mean over the data segment of the vertical position of the point at which the dye concentration is one half of its value in the mixed layer. 

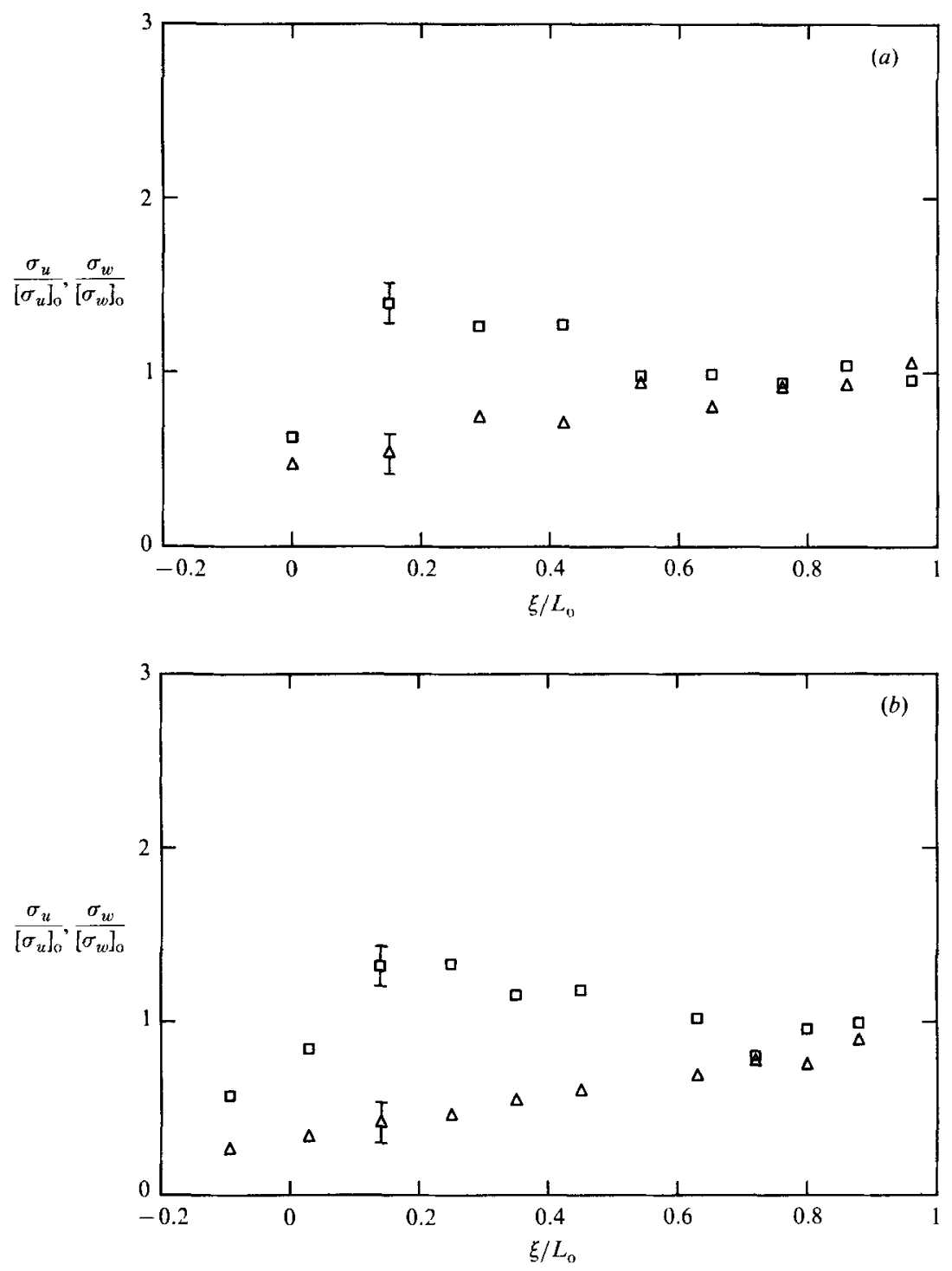

Figure 7. Variation of non-dimensional horizontal and vertical r.m.s. velocities with the nondimensional distance away from the interface, $\xi / L_{\circ} . \square, \sigma_{u} /\left[\sigma_{u}\right]_{0} ; \triangle, \sigma_{v} /\left[\sigma_{w}\right]_{0^{*}}(a) R_{j}=70 ;(b)$ $R_{j}=100$.

significant influence on the large eddies. In figure $8(b)$ the energy spectra for the horizontal velocity fluctuations indicate that there is a net energy gain at the lowfrequency ends of the spectra (large scales) as the density interface is approached. Moreover, the high-frequency ends of all spectra remain virtually unaffected, implying that the net transfer of turbulent kinetic energy from the vertical to the horizontal components of velocity is confined to small frequencies or large eddy scales.

It is of interest to see how the profiles of both vertical and horizontal turbulence intensities affect the distribution of the turbulent kinetic energy near the density interface. If we plot the total turbulent kinetic energy per unit mass, $\overline{q^{2}} /\left[\overline{q_{2}}\right]_{0}$, against 

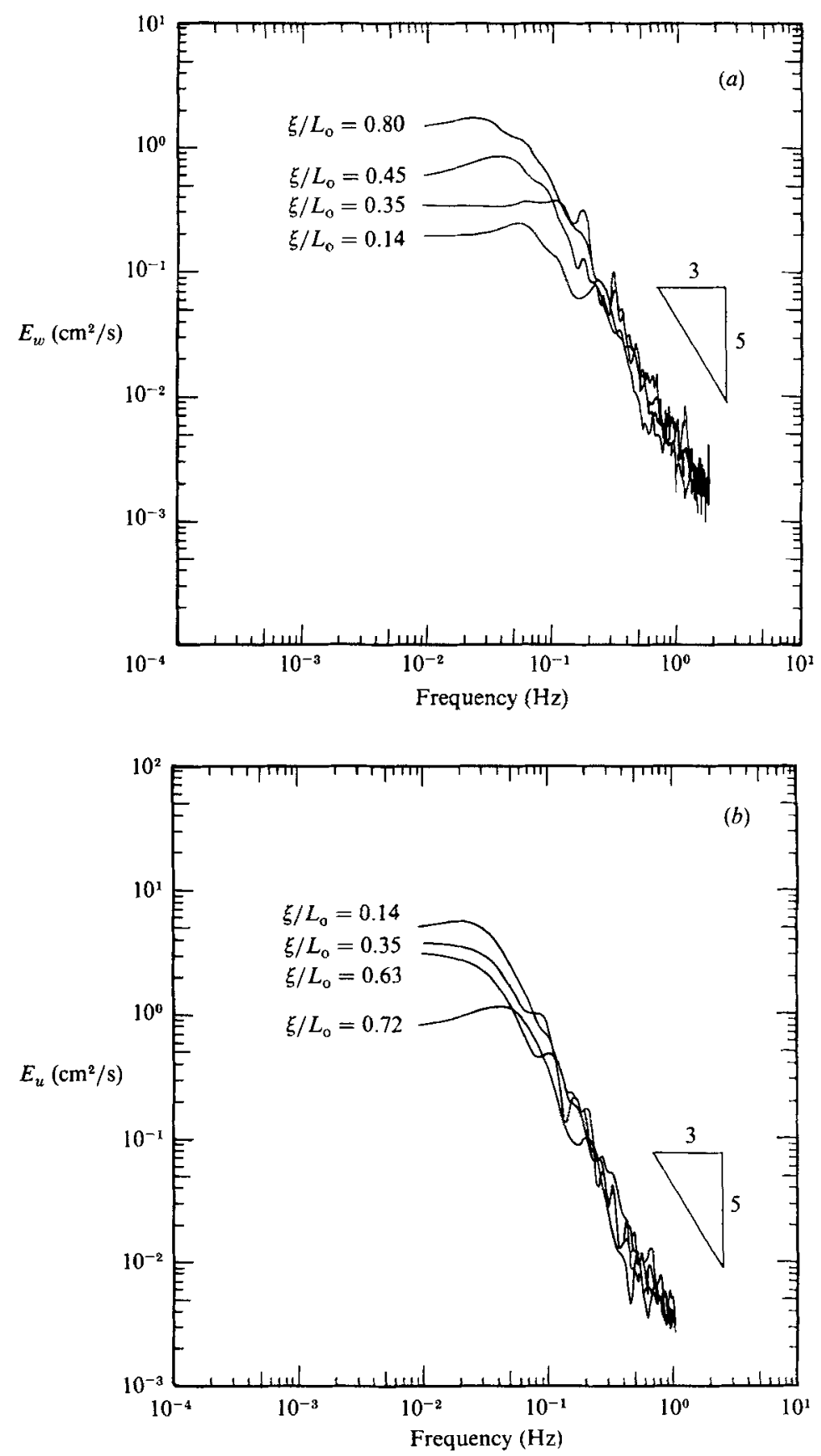

FrguRe 8. Energy spectra for the motion near a density interface. Spectra are for various values of $\xi / L_{0} . R_{j}=70$. (a) Vertical velocity fluctuations, $E_{w}$. (b) Horizontal velocity fluctuations, $E_{u}$,

$\xi / L_{0}$, as shown in figure 9 , we notice that the values of $\overline{q^{2}} /\left[\overline{q^{2}}\right]_{0}$ are less than 1 for $\xi / L_{\mathrm{o}}$ between about 0.5 and 1.0. A similar result was obtained by $H G$ for the case of shear-free turbulence near a rigid boundary. They explained the decrease in the energy of the turbulence as being due to a rise in the mean pressure. Very close to the flat plate HG predict that the energy of turbulence recovers to its value far from 


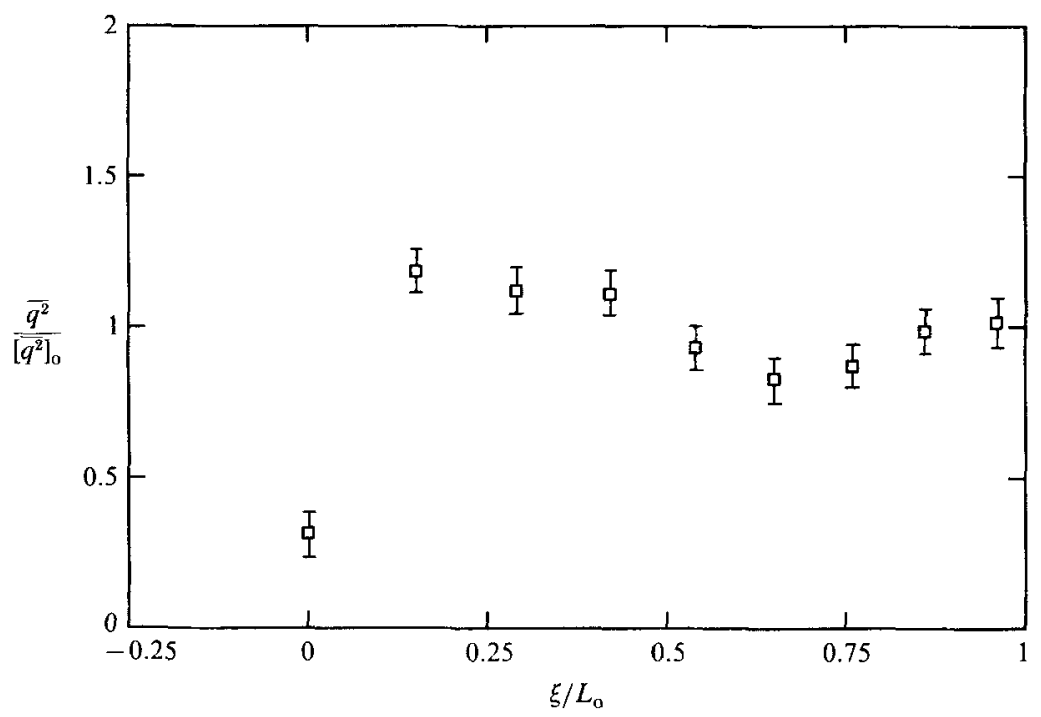

Figure 9. The variation of non-dimensional turbulent kinetic energy per unit mass, $\overline{q^{2}} /\left[\overline{q^{2}}\right]_{0}$, with non-dimensional distance from the mean position of the density interface, $\xi / L_{\mathrm{o}} . R_{j}=70$.

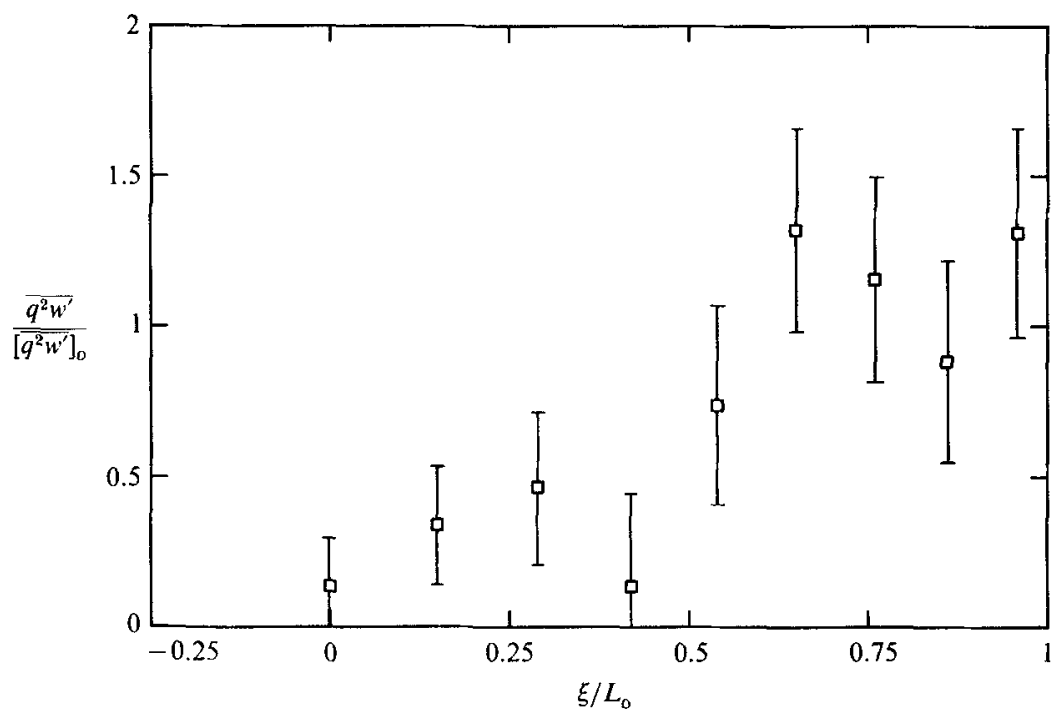

Figure 10. The variation of the non-dimensional vertical energy flux, $\overline{q^{2}} \overline{w^{\prime}} /\left[\overline{q^{2} w^{\prime}}\right]_{0}$, with the non-dimensional distance from the mean position of the density interface, $\xi / L_{0} . R_{j}=70$.

the boundary. Figure 9 shows that $\overline{q^{2}} /\left[\overline{q^{2}}\right]_{0}$ exceeds 1 near the density interface. This result is reproducible in every experiment and at first glance seems paradoxical owing to the fact that the turbulent kinetic energy near the interface exceeds its value in a homogeneous fluid. However, if we plot $\overline{q^{2} w^{\prime}} /\left[\overline{q^{2} w^{\prime}}\right]_{0}$ against $\xi / L_{0}$, we notice that $\overline{q^{2} w^{\prime}} /\left[\overline{q^{2} w^{\prime}}\right]_{0}$ drops from about 1 at $\xi / L_{\circ} \sim 1$, to zero as $\xi / L_{\circ}$ approaches zero as depicted in figure 10. This implies that the energy flux divergence increases and is consistent with the fact that the energy that was previously feeding the rest of the tank is now trapped in a layer of vertical dimension on the order of $L_{\mathrm{o}}$. As a result, 


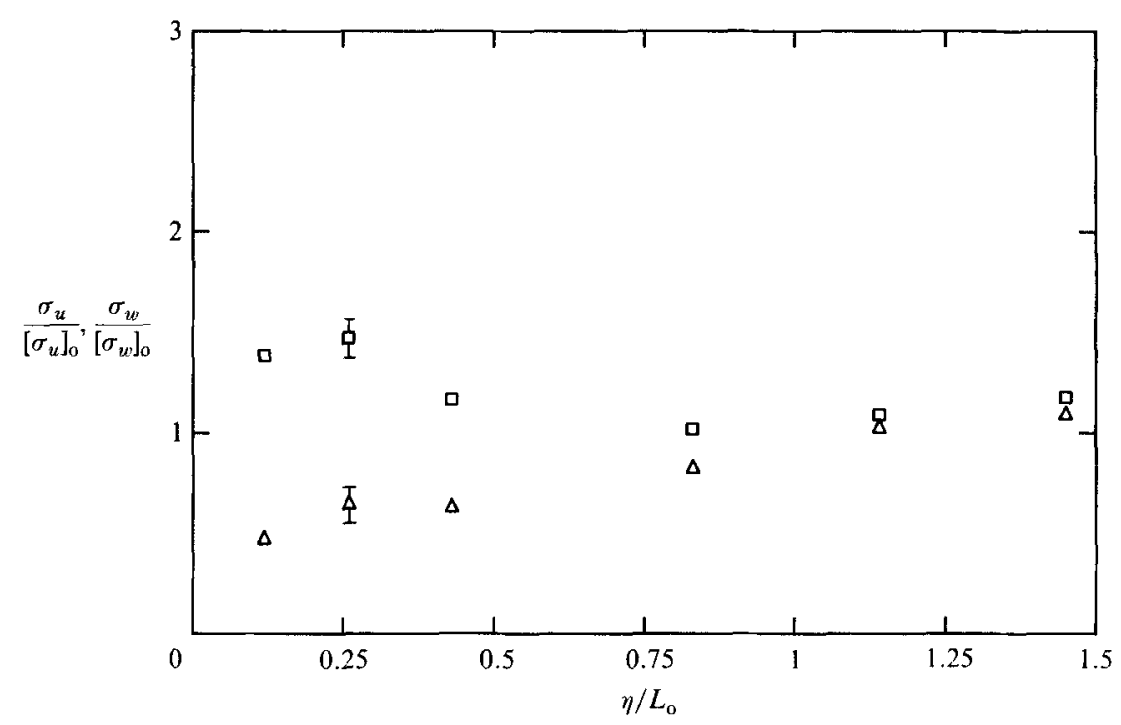

Figure 11. Variation of non-dimensional horizontal and vertical r.m.s. velocities with the nondimensional distance from the flat plate, $\eta / L_{0} . \square,\left[\sigma_{u}\right] /\left[\sigma_{u}\right]_{0} ; \triangle,\left[\sigma_{w}\right] /\left[\sigma_{w}\right]_{0}$.

turbulent kinetic energy values close to the interface may exceed those in a homogeneous fluid.

\subsection{Measurements near a rigid flat plate}

In order to compare the effect of a sharp density interface on shear-free turbulence to that of a rigid flat plate, we report in this section the results of velocity measurements made near a rigid flat plate. The same set-up was used as in the previous experiments except for the substitution of the two-fluid system with a homogeneous fluid and the insertion of a rigid flat plate in the flow. The details of the experimental techniques are described in $\$ 2.6$.

In figure 11 we plot $\left[\sigma_{u}\right] /\left[\sigma_{u}\right]_{0}$ and $\left[\sigma_{v y}\right] /\left[\sigma_{w}\right]_{0}$ against $\eta / L_{0}$ where $\eta$ is a vertical distance away from the flat plate and the subscript o refers to measurements in a homogeneous fluid in the absence of a flat plate at the same vertical location of the flat plate. Note that for this case, [] represents a spatial average of three measurements in the same horizontal plane along a straight line and at points separated by $2 \mathrm{~cm}$. The behaviour of both $\left[\sigma_{u}\right] /\left[\sigma_{u}\right]_{o}$ and $\left[\sigma_{w}\right] /\left[\sigma_{w}\right]_{o}$ shows a striking qualitative similarity to the experiments where a density interface is present. $\left[\sigma_{u}\right] /\left[\sigma_{u}\right]_{0}$ is amplified at small values of $\eta / L_{\mathrm{o}}$ while $\left[\sigma_{w}\right] /\left[\sigma_{w}\right]_{0}$ shows a continuous decay as we approach the rigid surface. Again, the effect of the wall on the turbulence seems to extend up to $\eta / L_{0} \sim 1$.

Figure $12(a)$ presents the energy spectra $E_{w}$ for the vertical velocity fluctuations at different distances $\eta / L_{0}$ away from the plate, whereas figure $12(b)$ shows the energy spectra $E_{u}$ for the horizontal velocity fluctuations. We notice that the flat plate is most effective at transferring energy from the vertical component to the horizontal components at small frequencies. The high-frequency end of the energy spectrum is virtually unaffected by the presence of the plate. This is equivalent to saying that larger eddies are most affected by the presence of the plate.

Figure 13 depicts the variation of $\left[\overline{q^{2}}\right] /\left[\overline{q^{2}}\right]_{0}$ vs. $\eta / L_{0}$ when the flat plate is present. On the same plot the variation of $\left[\overline{q^{2}}\right] /\left[q^{2}\right]_{0}$ in a homogeneous fluid in the absence of 

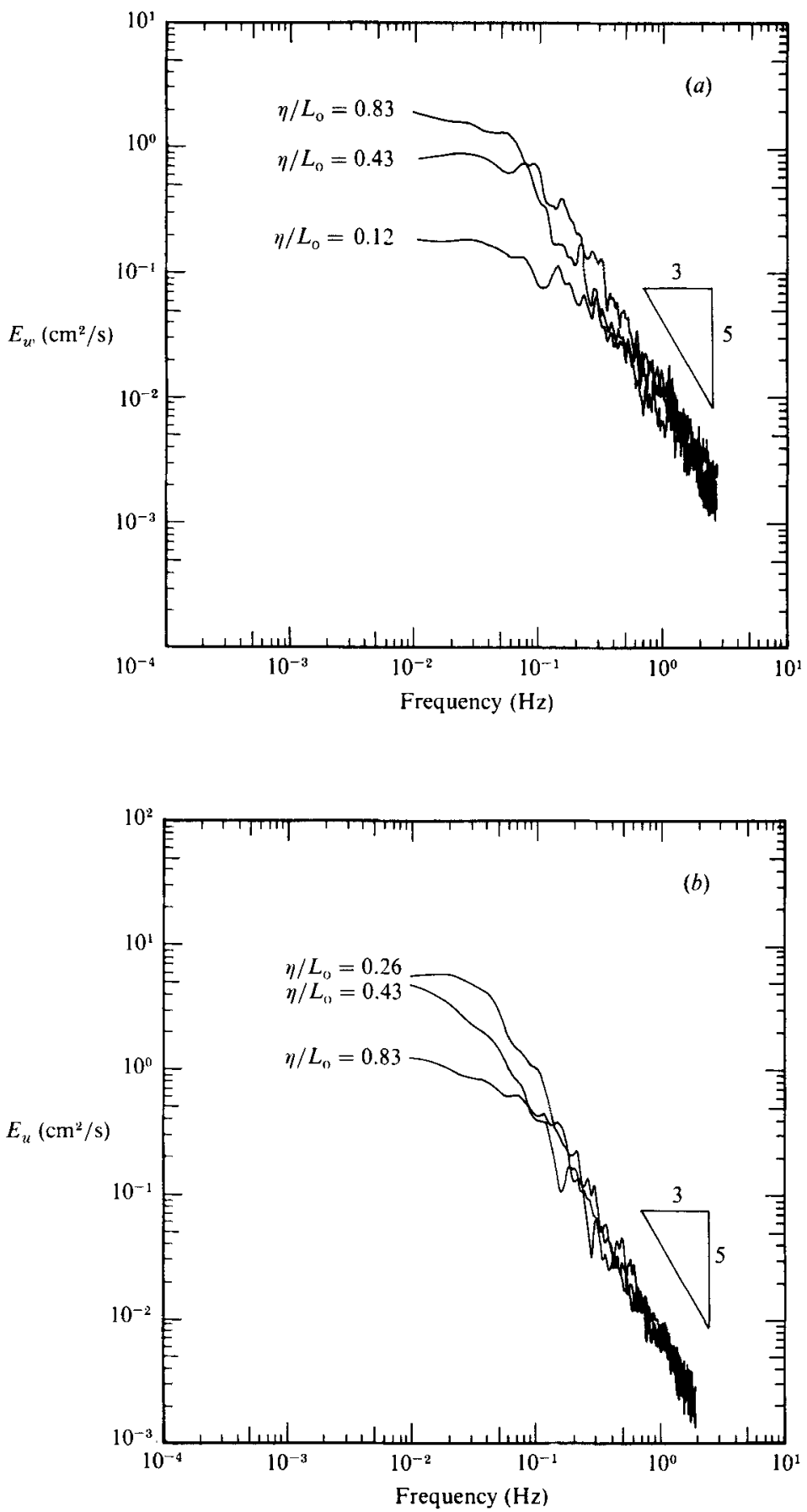

Figure 12. Energy spectra for the motion near a rigid flat plate. Spectra are for various values of $\eta / L_{0}$. (a) Vertical velocity fluctuations, $E_{w}$. (b) Horizontal velocity fluctuations, $E_{u}$ 


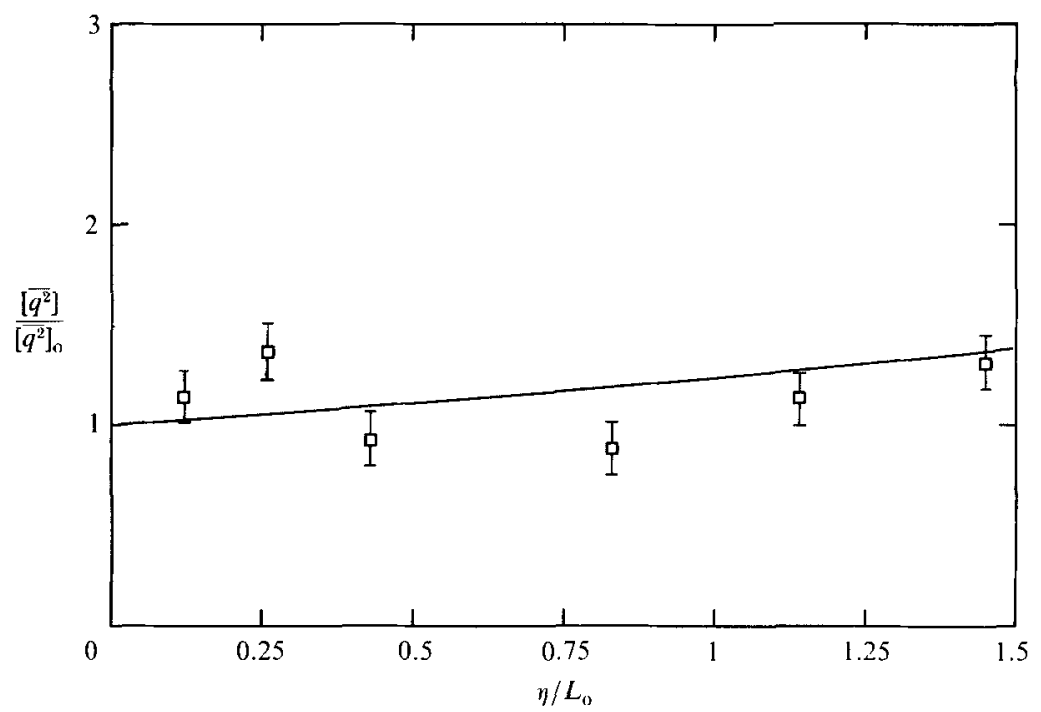

Figure 13. The variation of non-dimensional turbulent kinetic energy, $\left[\overline{q^{2}}\right] /\left[\overline{q^{2}}\right]_{0}$, with nondimensional distance from the flat plate, $\eta / L_{0}$. $\square$, measurements near a rigid flat plate. measurements in the absence of a flat plate. Curve is best fit to experimental data and is of the form $\left[\overline{q^{2}}\right] \sim 1 / z^{2}$.

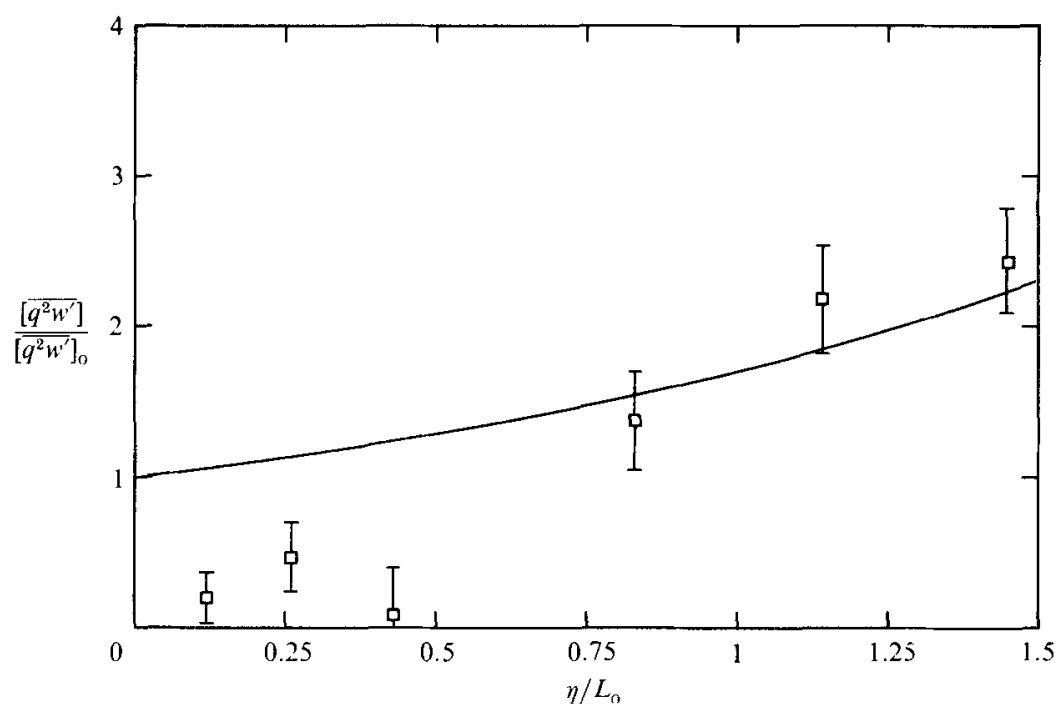

FiguRe 14. The variation of non-dimensional vertical energy flux, $\left[\overline{q^{2} w^{\prime}}\right] /\left[\overline{q^{2} w^{\prime}}\right]_{0}$, with nondimensional distance from the flat plate, $\eta / L_{0} . \square$, measurements near a rigid flat plate.

measurements in the absence of a flat plate. Curve is best fit to the experimental data (near the region where the flat plate was inserted) and is of the form $\left[\overline{q^{2} w^{\prime}}\right] \sim 1 / z^{3}$.

the plate is also shown $\dagger$. We notice that $\left[\overline{q^{2}}\right] /\left[\overline{q^{2}}\right]_{0}$ in the presence of a flat plate exceeds its value in the absence of a plate at small $\eta / L_{0}$. This result is also consistent with the increased values of the energy flux divergence near the rigid flat plate. Figure 14 presents $\left[\overline{q^{2} w^{\prime}}\right] /\left[\overline{q^{2} w^{\prime}}\right]_{0}$ plotted against $\eta / L_{0}$. It can be seen that

$\dagger$ It is to be noted that in figures 13 and $14\left[\overline{q^{2}}\right]$ and $\left[\overline{q^{2} w^{\prime}}\right]$ are normalized by their values in a homogeneous fluid at the plate and not by their values in a homogeneous fluid at the same location. Hence $\left[\overline{q^{2}}\right] /\left[\overline{q^{2}}\right]_{0}$ and $\left[\overline{q^{2} w^{\prime}}\right] /\left[q^{2} w^{\prime}\right]_{0}$ in a homogeneous fluid are not equal to 1 . 
$\left[\overline{q^{2} w^{\prime}}\right] /\left[\overline{q^{2} w^{\prime}}\right]_{0}$ decreases from about unity to zero as $\eta / L_{\mathrm{o}}$ varies from one to zero. This implies that the energy flux divergence is high near the flat plate, and as a result, the turbulent kinetic energy near the flat plate can exceed its value in the absence of the plate at the same location. From figure 13, we also notice that the turbulent kinetic energy per unit mass at about $\eta / L_{0}=0.5-1.4$ is less than that in a homogeneous fluid at the same distance from the grid in the absence of the plate. This might be associated with a rise in the mean pressure in that region as discussed by $\mathrm{HG}$ for shear-free turbulence near a rigid boundary.

\section{Discussion}

The primary objective of the present investigation was to study the nature of shear-free turbulence near a stable density interface and to acquire an increased understanding of turbulent mixing in stratified flows. The experiments indicate that the density interface acts, at least qualitatively, in a similar manner to a rigid flat plate where $\sigma_{u}$ is amplified, and $\sigma_{w}$ is sharply attenuated, in a layer adjacent to the plate. This can be regarded as a result of the flattening of the eddies near the interface/flat plate. The point at which the maximum amplification in $\sigma_{u}$ occurs is about $0.1-0.2 L_{\mathrm{o}}$ away from the interface or the flat plate. The effect of the interface/ flat plate on the turbulence seems to extend about one integral lengthscale.

Another point which this study considered was the determination of the turbulence scales that are most affected by the anisotropy created by either the density interface or the flat plate. The energy spectrum of the vertical velocity fluctuations shows that there is preferential attenuation of low frequencies (large scales) near the plate or the interface, indicating that the density interface/flat plate is efficient in damping large eddies. On the other hand, the energy spectrum for horizontal velocity fluctuations indicates that there is a substantial gain at the low-frequency end of the spectrum. In both the horizontal and vertical spectra, the high-frequency end was not significantly affected (since the velocity at the flat plate is zero the energy spectrum will eventually approach zero in a very thin viscous layer near the wall where no measurements were made).

One reason why the density interface affects the turbulence in a very similar manner to the rigid flat plate and flattens the turbulent eddies may be the sharpness of the density interface. Figure $15(a-c)$ presents pictures of the density interface taken by illuminating the flow with a sheet of laser light about $0.5 \mathrm{~mm}$ thick and with fluorescent dye added to the upper (mixed) layer. The advantage of this method of planar illumination is the preservation of the flow detail that is masked when the visualization method averages over a large horizontal section. Figure 15(a,b) indicates that the interface between the turbulent and the non-turbulent fluid can be very sharp. This is in contrast to the findings of Crapper \& Linden (1974), who visualized the flow through a shadowgraph and determined the interfacial thickness with a conductivity probe. It is thought that the determination of the interfacial layer thickness using a shadowgraph can be misleading since the resulting image represents an average over a large horizontal span. Detailed interfacial layer thickness measurements using laser-induced fluorescence techniques already completed support the fact that the interfacial layer thickness $h$ is finite but can be much smaller than $h / L_{\mathrm{o}} \sim 1-1.5$ as determined by Crapper \& Linden (1974). The full results of that investigation will be presented in a separate manuscript.

Turner (1968) concluded that the large eddies are entraining fluid in the form of sheets, which are ultimately mixed through the whole of the stirred layer. Although 

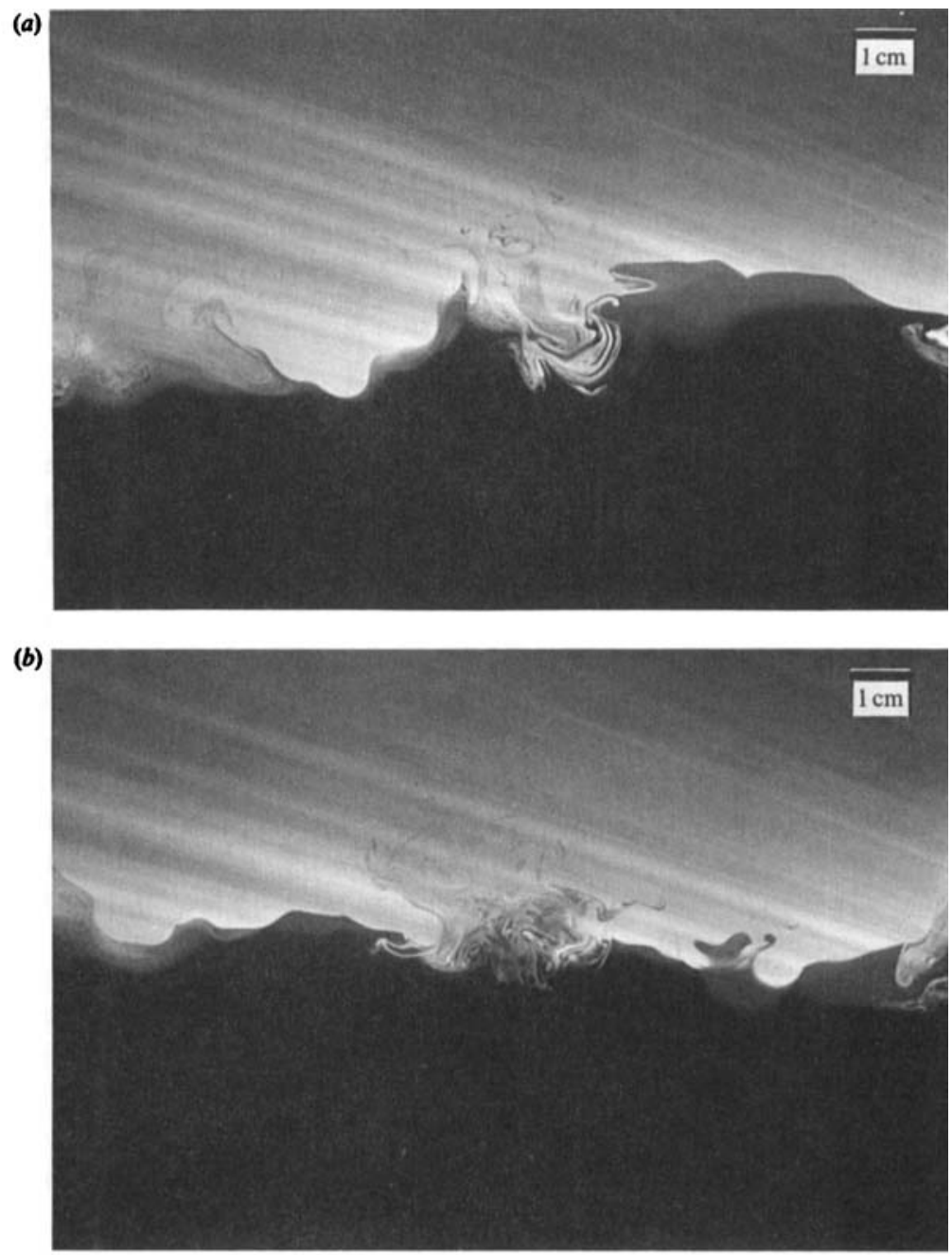

(c)

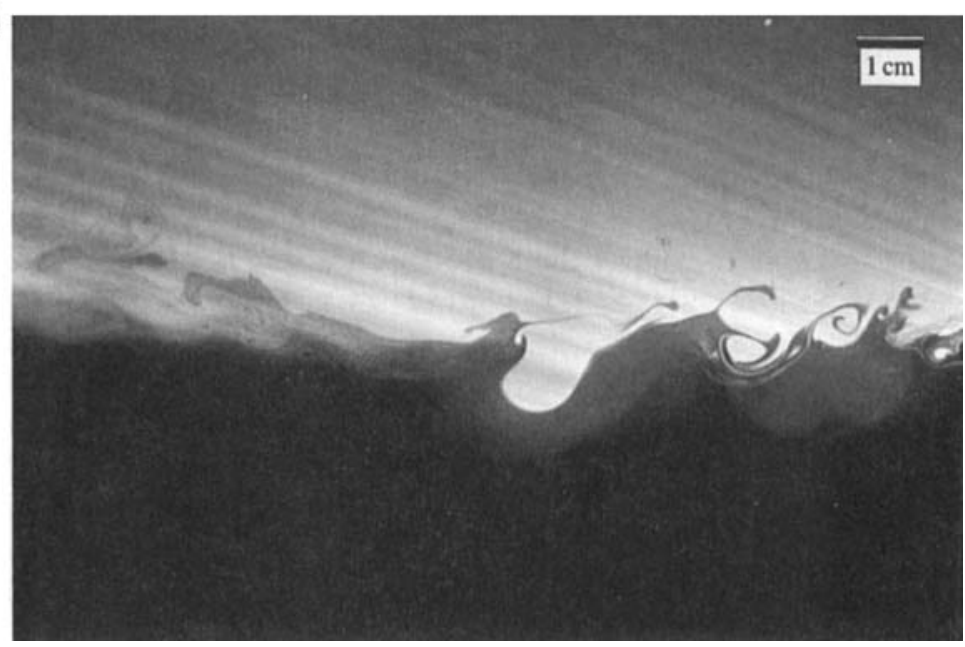

FigURE 15. Flow visualization using a thin sheet of laser light. The fluorescent dye is pre-mixed with the upper layer fluid which appears light. $R_{\tilde{j}} \sim 60$. 
our figures $15 a$ and $15 b$ are similar to Turner's figure $2(a)$ in that they show eddies impinging on the interface, cine films obtained in our experiment (using the same method of visualization) indicate that the large eddies that impinge on the interface 'bounce' back up with no significant entrainment of unstirred fluid. The films also indicate that patches of turbulence such as those in figures $15(a)$ and $15(b)$ might be the result of local interfacial instabilities that give rise to patches of intense, smallscale turbulence. Such patches occur sporadically both in time and space. Figure $15(c)$ also shows some intermittent mixing processes at a density interface. In this figure the mixing processes at the right of the picture seem to be more regular than those in figures $15(a)$ and $15(b)$ and have some characteristics of Kelvin-Helmholtz billows. It is worth noting that the occurrence of mixing events such as those in figure $15(c)$ is much less frequent than mixing events depicted in figures $15(a)$ and $15(b)$.

The findings of our investigation for both the density interface and the rigid flat plate indicate strong qualitative (and sometimes quantitative) agreement with the postulates of the $\mathrm{HG}$ theory. In particular, the amplification of horizontal velocities accompanied by a sharp reduction in the vertical velocity, the form of the energy spectra, and the distance up to which the wall/density interface effects are apparent are all in accordance with the predictions of the HG theory. At this point, it is worth noting that the $\mathrm{HG}$ theory is valid when the inertial effects of the turbulence are not important, and in the absence of a vertical energy flux. However, Hunt (1984) shows that the bssic conclusions of $H G$ are still valid even for $t>\tau_{0}$ provided the rate of dissipation of turbulent kinetic energy is constant for $\eta>L_{0}$. This might explain why our experiments which describe the steady-state flow near the plate show some agreement with the $\mathrm{HG}$ results. One observation which is not in good agreement with the HG results is the magnitude of the amplification of the horizontal turbulent velocity. Near both the density interface and the flat plate, $\sigma_{u}$ is amplified by as much as $40 \%$ as opposed to a maximum of $22 \%$ predicted by $\mathrm{HG}$. This discrepancy might be due to the increased values of the energy flux divergence near the density interface and the flat plate. Moreover, the decrease in the energy of the turbulence (compared to homogeneous fluid values) in a region near the interface and the flat plate is in qualitative agreement with $\mathrm{HG}$. They argued that the decrease in the energy of the turbulence is associated with a rise in the mean pressure in the same region.

The thickness of the viscous sublayer $\delta^{\nu}$, where the horizontal velocity begins to be attenuated, is predicted by HG to be $2\left(\nu \tau_{\infty}\right)^{\frac{1}{2}}$, where $\tau_{\infty}$ is the integral timescale of the turbulence in the free stream. This prediction holds for times on the order of $\tau_{\infty}$ after the turbulence encounters the wall. In our experiments, $2\left(v \tau_{0}\right)^{\frac{1}{2}}$ is approximately $0.35 \mathrm{~cm}$. The closest point to the plate at which we were able to measure velocities was $0.25 \mathrm{~cm}$ away from the plate surface. At that point $\left[\sigma_{u}\right]$ did not show any significant decay. Although we did not measure $\delta^{\nu}$ we can still suggest that $\delta^{v}$ in our experiments is not larger than predicted by a $2\left(\nu \tau_{0}\right)^{\frac{1}{2}}$ relationship.

Our results are also in agreement with some of the results in the model proposed by Long (1978). In that model, Long conjectures that the results of the theory of HG apply to the case where the rigid plate is replaced by a high $R_{j}$ density interface. Long also finds that the large turbulent eddies are flattened near the density interface and he characterizes the entrainment process as an intermittent bursting phenomena. Our results and flow-visualization pictures seem to support these arguments.

Although McDougall (1979b) also carried out experiments to measure the 
velocities in and near a density interface and in the vicinity of a rigid flat plate, his results did not indicate any substantial amplification of the horizontal velocity in the vicinity of the flat plate. According to McDougall there is a 'hint of some amplification of the horizontal velocity' at higher $R_{i}$ but he did 'not have enough accurate data to assert this with confidence'. This observation might possibly be due to the low Reynolds numbers used in McDougall's experiments. The Reynolds number $R e=\left[\sigma_{w}\right]^{2}[\tau] / \nu$ for his experiments was about 40 , as opposed to values of around 120 in our experiments. The HG theory is valid only at high Reynolds numbers and the experiments of Uzkan \& Reynolds (1967) and Thomas \& Hancock (1977) clearly show that. They also demonstrate that high-Reynolds-number turbulence is necessary for the amplification of the horizontal velocity component.

In summary, it is apparent that the behaviour of oscillating-grid-induced turbulence near a sharp density interface apparently has a great deal of similarity to that near a solid boundary. The essential features of the motion are remarkably close in nature and are described reasonably well by the theory of Hunt \& Graham (1978).

The authors would like to thank Dr James Skjelbreia for help throughout this work and Dr D. Papantoniou for setting up the data acquisition system used in the concentration measurements. We would also like to thank Dr. P. Papanicolaou and the staff of the W. M. Keck Hydraulics Laboratory for their assistance in the construction of the equipment. The work was supported by the National Science Foundation Grant MSM 84-12641, A01. During part of this study, H.J.S.F. was supported by NSF Grant MSM 8504909.

\section{REFERENCES}

Crapper, P. E. \& Linden, P. F. 1974 The structure of turbulent density interfaces. J. Fluid Mech. 65,45 .

Fernando, H. J. S. \& Long, R. R. 1983 The growth of a grid-generated turbulent mixed layer in a two-fluid system. J. Fluid Mech. 133, 377 .

Fernando, H. J. S. \& Long, R. R. 1985 a Mixed-layer growth in stratified fluids. In The Ocean Surface: Wave Breaking, Turbulent Mixing and Radio Probing (ed. Y. Toba \& H. Mitsuyasu), p. 541 .

Fernando, J. J.S. \& Long, R. R. $1985 b$ On the nature of the entrainment interface of a twolayer fluid subjected to zero-mean shear turbulence. J. Fluid Mech. 151, 21.

Fernando, H. J. S. \& Long, R. R. $1985 c$ The growth of a shear-free mixed layer in a linearly stratified fluid. Phys. Fluids 28, 2999.

Folse, R. F., Cox, T. P. \& Schexnayder, K. R. 1981 Measurements of the growth of a turbulently mixed layer in a linearly stratified fluid. Phys. Fluids. 24, 396.

Gartrell, G. 1979 A signal processor for a laser-Doppler velocimeter. W.M. Keck Laboratory of Hydraulics and Water Resources Tech. Mem. 78-5. California Institute of Technology.

Hannoun, I. A. 1985 Matching the refractive index in density stratified flows. W.M. Keck Laboratory of Hydraulics and Water Resources Tech. Mem. 85-1. California Institute of Technology.

Hopfinger, E. J. \& Toly, J. A. 1976 Spatially decaying turbulence and its relation to mixing across density interfaces. $J$. Fluid Mech. 78, 155.

Hopfinger, E. J. \& Linden, P. F. 1982 Formation of thermoclines in zero-mean shear turbulence subjected to a stabilizing buoyancy flux. J. Fluid Mech. 114, 157.

Hunt, J. C. R. 1984 Turbulence structure in thermal convection and shear-free boundary layers. J. Fluid Mech. 138, 161.

Hunt, J. C. R. \& Graham, J. M. R. 1978 Free-stream turbulence near plane boundaries. J. Fluid Mech. 84, 209. 
Kitaigorodski, S.A. 1979 Review of the theories of wind-mixed layer deepening. Marine Forecasting, Elsevier Oceanographic Ser. 25, 1.

Koochesfahani, M. M. 1984 Experiments on turbulent mixing and chemical reaction in a liquid mixing layer. Ph.D. thesis. California Institute of Technology.

LINDEN, P. F. 1975 The deepening of a mixed layer in a stratified fluid. J. Fluid Mech. 78, 385.

Lru, H. T., Lin, J. T., Delisi, D. P. \& Robben, F. A. 1977 Application of a fluorescence technique to dye-concentration measurements in a turbulent jet. NBS Special Publication 484 . in Proc. Symp. on Flow in Open Channels and Closed Conduits held at NBS, Gaithersburg, MD, February 1977.

LoNG, R. R. 1978 A theory of mixing in stably stratified fluids. J. Fluid Mech. 84, 113.

McDougall, T. J. $1979 a$ On the elimination of refractive index variations in turbulent density stratified flows. $J$. Fluid Mech. 93, 83.

MCDougaLL, T. J. $1979 b$ Measurements of turbulence in a zero-mean shear mixed layer. J. Fluid Mech. 94, 409.

PAPANICOlaOU, P. N. 1985 Mass and momentum transport in a turbulent buoyant vertical axisymmetric jet. Ph.D thesis. California Institute of Technology.

Papantoniov, D. P. 1986 Observations in turbulent buoyant jets by use of laser-induced fluorescence. Ph.D. thesis. California Institute of Technology.

PhilLIPs, O. M. 1977 Dynamics of the Upper Ocean. Cambridge University Press.

Tennekes, H. 1975 Eulerian and Lagrangian time microscales in isotropic turbulence. J. Fluid Mech. 67, 349 .

Thomas, N. H. \& Hancock, P. E. 1977 Grid turbulence near a moving wall. J. Fluid Mech. 82, 97.

Thompson, S. M. \& TuRner, J. S. 1975 Mixing at an interface due to turbulence generated by an oscillating grid. J. Fluid Mech. 67, 349.

TURNer, J.S. 1968 The influence of molecular diffusivity on turbulent entrainment across a density interface. J. Fluid Mech. 33, 639.

Turner, J. S. 1979 Buoyancy Effects in Fluids. Cambridge University Press.

Uzkan, T. \& Reynolds, W. C. 1967 A shear-free turbulent boundary layer. J. Fluid Mech. 28, 803.

WANG, J. \& WU, J. 1985 Wind induced water turbulence. In The Ocean Surface: Wave Breaking, Turbulent Mixing and Radio Probing. (ed. Y. Toba \& H. Mitsuyasu), p. 401. 